\title{
INTERSECCIÓN DE LA ESPIRITUALIDAD/RELIGIÓN EN LAS CONDUCTAS SEXUALES DE LOS/AS ADOLESCENTES
}

\author{
Intersection of spirituality/religion on the adolescents' sexual behavior
}

LOURDES I. Morales AlejANDro ${ }^{1}$

\section{Resumen}

Este artículo revela los hallazgos de un estudio exploratorio con un diseño no experimental de tipo transversal correlacional que examina la influencia de la espiritualidad y la religión en las conductas sexuales de los/as adolescentes puertorriqueños/as. Se desarrolló el Instrumento para Evaluar la Influencia de la Religiosidad en las Conductas Sexuales: Versión Lourdes Morales Alejandro (IEIRCS: VLMA), cuyo contenido fue evaluado por nueve expertos en trabajo social, religión/ espiritualidad y construcción de pruebas. Los análisis cuantitativos se efectuaron utilizando un nivel alfa de.05. El análisis psicométrico fue conducido con rotación Varimax, resultando un instrumento con 21 reactivos y cinco componentes. La validez de contenido alcanzó un Alfa Cronbach de.848. Fue administrado a 251 participantes, de 15 a 18 años, en siete escuelas públicas puertorriqueñas de la Región Metropolitana de San Juan. Todos seleccionados por disponibilidad, con el consentimiento de sus padres.

Los hallazgos respaldan el rol de la religión como factor proscriptor e inhibidor de las relaciones sexuales premaritales. Se encontraron diferencias estadísticamente significativas $(p<.05)$ entre católicos y protestantes/evangélicos en cuanto a la influencia de la religión en sus conductas sexuales. Los/as protestantes parecen ser más conservadores respecto a involucrarse en relaciones sexuales prematrimoniales. Se observó que, a pesar del compromiso de ambos grupos con sus creencias religiosas, este es insuficiente para impedirles involucrarse en algún tipo de actividad sexual. Este estudio contribuye a la producción de conocimiento sobre el tema a través de un instrumento validado y fomenta su integración en la práctica profesional del trabajo social.

Palabras clave: Adolescentes; Conducta Sexual; Religión; Espiritualidad; Adolescentes Cristianos

\begin{abstract}
This article reveals the findings of an exploratory study with a correlational non-experimental cross-sectional design to examine the influence of spirituality and religion on the sexual behaviors of Puerto Rican adolescents. An instrument was developed to assess the Influence of Religiosity on Sexual Behaviors; Version Lourdes Morales Alejandro (IEIRCS: VLMA). Nine experts in social work, religion/spirituality and test construction, assessed the content. All quantitative analysis of the instrument was done using an alpha of 0.05. Psychometric analysis with Varimax rotation was performed, leaving the instrument with 21 items and five components. The content validity reached a Cronbach Alpha of.848.

The instrument was administered to 251 participants, 15 to 18 years old, in seven public schools in the metropolitan area of San Juan, Puerto Rico. The participants were selected by availability, with the consent of their parents.

The results of the study support the role of religion as a proscriptive factor and inhibitor of sexual intercourse before marriage. Statistically significant differences ( $\mathrm{p}<.05$ ) between Catholics and Protestants/ Evangelicals, regarding the influence of religion on their sexual behaviors, were found. Protestants seem to be more conservative about engaging in sex before marriage. It was noted that, despite the commitment of both groups to their religious beliefs, this is not enough to prevent them from getting involved in any sexual activity. This study contributes to the production of knowledge on the subject through a validated instrument and promotes its integration into the professional social work practice.
\end{abstract}

Keywords: Adolescents; Sexual Conduct; Religion; Spirituality; Christian Adolescents

Recibido: 27/10/2014

Aceptado: 25/11/2014

Publicado: 01/12/2014

1. Catedrática Asociada. Departamento de Trabajo Social, Universidad de Puerto Rico. Río Piedras, Puerto Rico. Dra. Lourdes I. Morales Alejandro. P. O. Box 1645. Guaynabo, Puerto Rico 00970. lourdes.morales1@upr.edu; research.dr.moralesalejandro@gmail.com 


\section{Introducción}

Múltiples estudios científicos han vinculado la espiritualidad y la religión con la conducta de los/as adolescentes, especialmente, como factor protector de la conducta sexual temprana (Barret, 2003; Knight, et al., 2007; Sinha, Cnaan y Gelles, 2007). A estas se les adjudica influenciar directamente en la decisión de posponer, reducir y evitar la primera relación sexual (Davidson, Moore, Earle y Davis, 2008; Farmer, Trapnell y Meston, 2008; Hardy y Raffaelli, 2003; Rotosky, Regnerus y Comer, 2003). Se ha encontrado que los/as jóvenes que dan mayor importancia a la religión y asisten más frecuentemente a los servicios religiosos, tienen actitudes más restrictivas hacia el sexo premarital, reportan menos experiencia sexual, son más propensos a retrasar las relaciones sexuales y a tener menos parejas sexuales (Haglund y Fehring, 2010; Remez, 1990). Sin embargo, existe evidencia científica que revela el comportamiento sexual «liberal» de los/as adolescentes religiosos/as (Burdette y Hill, 2009; Uecker, Angotti y Renerur, 2008), poniendo en tela de juicio el control real de las creencias religiosas y espirituales en los/as adolescentes y consiguientes consecuencias emocionales y espirituales.

Existe un amplio desconocimiento del/la profesional de trabajo social y otros/as profesionales de la conducta y de la salud sobre la espiritualidad y la religiosidad, y por consiguiente, limitadas destrezas para integrarlas a la intervención profesional (Hugen, 2001; Hutchinson, 2008; Peo Early, 1998; Walsh, 2003). Dos de las principales razones para ello se centran en la percepción de que son un asunto personal y privado, por lo tanto controversial (Hutchison, 2008: 11); y su no inclusión en el currículo subgraduado y graduado en los programas académicos universitarios que preparan y desarrollan a los/as trabajadores/as sociales puertorriqueños/as. Esto, a pesar del compromiso ético del servicio holístico de la persona en el ambiente, la sensibilidad a la diversidad cultural y la misión profesional de promover la satisfacción de las necesidades básicas, el bienestar y la justicia para todos los individuos y comunidades.

Puerto Rico es un país históricamente marcado por la religión. Desde la perspectiva espiritual, existe una amplia diversidad de filosofías espirituales y religiones que facilitan la selección a la carta de cualquiera de ellas de forma personalizada. La religión cristiana es de mayor predominancia, en particular, la denominación católica y protestante. Los/as adolescentes puertorriqueños/ as están socializados/as en un contexto religioso, aunque en el hogar no haya una religión o afiliación religiosa definida. Así, la religión posee un rol importante en moderar las conductas sexuales de los/as niños/as y adolescentes puertorriqueños/as, por lo que estudiar este fenómeno adquiere gran 
relevancia socio-psicológica. No obstante, existe una marcada escasez de estudios científicos que relacionen la sexualidad con la religión en la población de niños/as y adolescentes, y el papel que desempeña en la prevención de las conductas de riesgo.

La abundante evidencia científica que revela la liberalización del comportamiento sexual de los/as adolescentes religiosos/as y la carencia de evidencia empírica sobre la relación de la espiritualidad, la religión y las conductas sexuales de los/as adolescentes puertorriqueños motivó a la investigadora a desarrollar un instrumento que permitiera evaluar esta relación y una vez validado, obtener los datos necesarios que permitieran examinarla. Desde esta perspectiva, los propósitos del estudio fueron: (a) examinar la influencia de la espiritualidad y la religiosidad en las conductas sexuales de los/las adolescentes religiosos/as y no religiosos/as; (b) determinar si los/las adolescentes religiosos/as se involucraban en actividades sexuales sustitutivas de la relación coital; y (c) examinar la relación del género con las conductas y actividades sexuales de los/las adolescentes. Se seleccionó un escenario totalmente secular y con estrictas estipulaciones de separación iglesia y estado con miras a tener amplia representatividad de creencias hacia la religión y la espiritualidad.

\section{Revisión de literatura}

Los conceptos espiritualidad y religión son vistos por muchos como sinónimos y por otros, como inseparables (Canda, 1988; Hugen, 2001), o por lo menos que existe una frontera poco clara entre ellos. Espiritualidad es definida como aquellos aspectos de los sentimientos del individuo, aspiraciones y necesidades que están relacionados a los esfuerzos del ser humano por encontrar propósito y significado a las experiencias de la vida, los cuales no tienen que estar atados a un cuerpo eclesiástico de creencias y prácticas organizadas (Becvar, 1998; Hugen, 2001). Religión es un sistema de creencias y prácticas observadas por una comunidad, apoyada por rituales que reconocen, adoran y comunican con o se dirigen a lo sagrado, a lo trascendental (Koenig, 2008; Pargament, 1997). Provee un código de conducta al que los miembros intentan adherirse (Hutchison, 2008). La función más importante de la religión es espiritual (Pargament, 1997) pero no toda espiritualidad es religiosa (Compte-Sponville, 2006).

La religión está tomando el rol de control social a través de la alianza de las instituciones sociales iglesia y familia en una sociedad que ha disminuido su estigma hacia la maternidad fuera del matrimonio, glorifica el sexo en los medios de comunicación (radio, televisión, internet, videos musicales), y en la que los padres/madres muestran incapacidad para comunicarse efectivamente 
con sus hijos e hijas (Papalia, Wendkos Olds y Duskin Fieldman, 2010). El descarrío de los/as jóvenes de los estándares establecidos por estas dos instituciones sobre la conducta sexual, especialmente, cuando actúan como aliadas, trae como resultado sentimientos de culpa, vergüenza, amenaza, expectativa de castigo divino (Hardy y Rafaelli, 2003) y la percepción de la disminución del grado de espiritualidad, al alejarse o desconectarse de su Ser Supremo o Dios (Morales Alejandro, 2014). La contradicción entre lo que creen y cómo se comportan puede provocarles angustia y llevarlos a elegir desviarse de las normas religiosas. Así como inducirlos/as a la eliminación o disminución de la conducta o alterar sus creencias religiosas, incluyendo abandonarlas (Hardy y Rafaelli, 2003). Sin embargo, estos sentimientos contradictorios pueden variar cuando los/as adolescentes santifican las relaciones sexuales al visualizarlas como una manifestación directa de Dios (por ej. «Dios está presente en la unión sexual entre dos personas que se aman».) y le adscriben cualidades sagradas a la experiencia sexual (Murray-Swank, Pargament y Mahoney, 2005).

Murray-Swank, Pargament y Mahoney (2005: 213) realizaron un estudio sobre la santificación de las relaciones coitales en un grupo de 151 estudiantes universitarios (bachillerato) en Estados Unidos que, estuvieran involucrados en relaciones amorosas y relaciones sexuales no maritales. Encontraron que los/as estudiantes que percibieron las relaciones sexuales como sagradas, experimentaron gran satisfacción en ellas. La creencia de que Dios era parte de la relación sexual en una relación amorosa estuvo asociada a reacciones afectivas más positivas al intercambio sexual. Entre más percibieron características sagradas en su relación sexual no marital, más probabilidad hubo de que se comprometieran con ella y aumentó el número de relaciones de parejas duraderas.

La doctrina judeocristiana ubica las relaciones sexuales como sagradas, pero dentro del matrimonio. Promueve la abstinencia sexual como base de la pureza sexual en la que la virginidad, tanto en el hombre como en la mujer, simboliza el compromiso espiritual y conexión con Dios. El cuerpo humano, bajo esta doctrina, se percibe como templo del Espíritu Santo y lo que contriste al Espíritu es pecado y separa al/la cristiano/a de Dios. Por consiguiente, la expectativa de la iglesia y las familias religiosas y aún la del/la mismo/a joven, es que las relaciones amorosas integren el compromiso de privarse totalmente de satisfacer los apetitos sexuales hasta el matrimonio (abstinencia sexual) y llegar a él virgen.

Un estudio de Remez (1990: 42) reveló que la denominación religiosa influye las actitudes hacia el sexo premarital. Los hallazgos apuntaron a que 
los/as adolescentes con afiliaciones protestantes fundamentalistas, bautistas y judíos tuvieron actitudes menos permisivas hacia el sexo premarital que los/ as adolescentes de otras afiliaciones religiosas, entre ellas, la católica. Ogland e Hinojosa (2012: 425), confirmaron este hallazgo en un estudio con 2,000 brasileños (18 años de edad en adelante), quienes tendieron a emitir un juicio moral restrictivo del sexo antes del matrimonio y la cohabitación debido a un fuerte compromiso con la Biblia para interpretar las tendencias culturales y sociales.

Por otro lado, existe evidencia científica que indica que los/as adolescentes religiosos/as incurren en actividades sexuales a pesar de su compromiso religioso. Un estudio realizado por Burdette y Hill (2009: 42) con una muestra de 3,290 adolescentes estadounidenses, de 13 a 17 años de edad, en el que el $31 \%$ de los participantes eran miembros de grupos de protestantes conservadores, demostró que, a pesar de que la prominencia religiosa tuvo una asociación fuerte en la demora o retraso de las relaciones sexuales, estos/as se involucraban en otras actividades sexuales como el manoseo y el sexo oral. Las prácticas religiosas de los/las adolescentes de orar, rezar y leer la Biblia estuvieron fuertemente asociadas a la demora en su actividad sexual. El estudio también reveló que, no había relación entre asistir a la iglesia y practicar el sexo oral. Los investigadores atribuyen este hallazgo a que, las orientaciones sobre sexo recibidas por los/as jóvenes en sus respectivas iglesias no incluyen otras formas de actividad sexual. Esto permite que la influencia religiosa en la transición del/la adolescente al sexo oral sea menor a la de la relación sexual. Lo contrario sucedió con los/las adolescentes no religiosos/as. Las razones para estos evitar las actividades sexuales estuvieron fundamentadas en la exposición pública que tenían a los mensajes de salud sexual. Mensajes a los que los/las adolescentes religiosos/as podrían estar protegidos/as por ser percibidos como opuestos a su doctrina.

Uecker, Angotti y Renerur (2008: 1208) evaluaron la afirmación de que los/as jóvenes religiosos/as y los/as que se comprometieron con la abstinencia sexual (no relaciones vaginales) tenían una mayor probabilidad de involucrarse en otras actividades sexuales. Encontraron que las actividades sexuales sustitutivas de las relaciones sexuales vaginales se debían al alto énfasis que las instituciones religiosas ponían a la "pureza sexual», aspecto altamente influenciado por la cultura. Estos aducen que, por razones morales, no existe en los/las jóvenes una virginidad per se, sino una virginidad técnica. Esto es, un intercambio de los placeres de las relaciones sexuales por sustitutos de menor riesgo como lo son el sexo oral, la masturbación mutua, el uso de pornografía solitaria y la masturbación. De acuerdo con dichos investigadores, este «tipo 
de virginidad» es evidencia de un fenómeno naciente conocido como moral de clase media, en el que, mantener la virginidad no es por consecuencia de la religión ni por el compromiso de la abstención sexual, sino formas de mantener los planes escolares futuros y las oportunidades socioeconómicas.

De igual manera, las instituciones sociales de la familia y la escuela influyen las actitudes y conductas sexuales de los/as adolescentes. Estas instituciones, en particular la familia, conforman el sistema de orientación moral, espiritual y religioso de sus miembros. La conciencia de lo moral y las relaciones éticas con los demás se fundamentan en este sistema. El modelaje de los padres y madres es esencial en cuanto a lo que piensan respecto a las relaciones sexuales, el tiempo adecuado y las implicaciones en involucrarse en ellas.

\section{Materiales y Método}

Este estudio es exploratorio porque examina un tema poco estudiado en Puerto Rico en la población de adolescentes. El diseño es no experimental, transversal-correlacional. En él no se manipularon las variables, tampoco se expuso a los sujetos a condiciones o estímulos. Los datos se recolectaron en un tiempo específico, con el interés de analizar su incidencia e interrelación en el momento dado; además de describir las relaciones entre dos o más variables (Hernández Sampieri, Fernández Collado y Baptista Lucio, 2007).

A partir de la teoría y las investigaciones, se formularon las siguientes hipótesis: (a) existen diferencias estadísticamente significativas a un nivel de $p<.05$ en las conductas sexuales de los/as participantes religiosos/as y los/ as no religiosos/as; (b) existen diferencias estadísticamente significativas a un nivel de $p<.05$ entre los valores de la escala Influencia de la Espiritualidad en las Conductas Sexuales de los/as adolescentes religiosos/as y no religiosos/ as; (c) los/as adolescentes religiosos/as se involucran en actividades sexuales sustitutivas a la relación coital; y (d) existen diferencias significativas $(p<.05)$ por género en las conductas sexuales de los/as adolescentes religiosos/as y no religiosos/as.

\section{Participantes}

La población que se estudió fue la de adolescentes de 15 a 18 años de edad, de ambos sexos, de las escuelas públicas (estatales) de la Región Metropolitana de San Juan, del Departamento de Educación del Estado Libre Asociado de Puerto Rico. Se seleccionaron escuelas estatales por la diversidad de estudiantes que provienen de diversos municipios escolares, nivel socioeconómico, raza, etnia, religión y espiritualidad. El Departamento divide sus escuelas por 
regiones educativas, distritos y municipios. La región del estudio alberga 218 escuelas rurales y urbanas, de cinco distritos diferentes. De acuerdo al orden territorial, pertenecen a la región norte del país, adyacentes a San Juan, la capital. Los criterios de selección de las escuelas fueron: pertenecer a la región mencionada; nivel académico intermedio y superior; y tener una matrícula mínima de 300 estudiantes.

El Comité Institucional para la Protección de los Seres Humanos en la Investigación de la Universidad de Puerto Rico y la División de Investigaciones Pedagógicas, adscrita al Área de Planificación y Desarrollo Educativo del Departamento de Educación, evaluaron minuciosamente la propuesta de investigación y los documentos concernientes a la confidencialidad del proceso. Una vez se recibió la autorización de ambas instancias, el Departamento permitió la participación de las 16 escuelas que cumplieron con los requisitos establecidos. A su vez, la condicionó a solo los/as estudiantes que hubieran completado la asignatura Paternidad/Maternidad Responsable o la de Salud o ambas. Del total de las escuelas seleccionadas, catorce (14) ofrecían estos cursos. Seis escuelas urbanas, de cuatro distritos diferentes: Carolina, San Juan I, II y Guaynabo mostraron interés en participar; los dos primeros distritos con dos escuelas y los restantes con una escuela cada uno. El tamaño del universo muestral fue de 1,069 estudiantes.

El proceso de reclutamiento de los/as participantes se inició con la autorización y apoyo de los respectivos directores escolares, y la coordinación con los profesores, trabajadores sociales y consejeros de las escuelas participantes. Como producto de este proceso, 251 estudiantes participaron voluntariamente en la investigación. De estos, 156 (62.2\%) son mujeres y 95 (37.8\%) son varones. La mayoría de los/as participantes tenía 17 años de edad. El 95.9\% informó ser heterosexual, 2\% homosexual y 1.6\% bisexual. El $42.6 \%$ de los/ as participantes cursaban el undécimo grado y $57.0 \%$ el duodécimo. En ambos niveles académicos la denominación católica predominó: $23.9 \%$ y $28.7 \%$, respectivamente. La segunda fue la protestante/evangélica con $18 \%$ y $22.4 \%$, respectivamente.

El $45 \%$ residía con ambos padres y el $48.2 \%$ con la madre. Las féminas adolescentes fueron las que mayormente residían con sus madres. Los padres y las madres de los/as adolescentes habían obtenido algún grado universitario. El nivel académico más frecuente fue el bachillerato.

\section{Instrumento}

Los datos se recopilaron a través de un cuestionario redactado en español denominado: Instrumento para Evaluar la Influencia de la Religiosidad en las 
Conductas Sexuales: Versión Lourdes Morales Alejandro (IEIRCS: VLMA). Los reactivos de dicho cuestionario se elaboraron de acuerdo con la revisión de la literatura sobre el tema. Nueve jueces profesionales, expertos/as en trabajo social, religión/espiritualidad y construcción de pruebas, evaluaron el contenido (identificaron cuál ítem era esencial), siguiendo el procedimiento descrito por Lawshe (1975), quedando finalmente conformado por 95 reactivos. Los reactivos que obtuvieron una puntuación menor de 0.70 fueron eliminados. El cuestionario se contestó a través de una escala de respuestas tipo Likert de cuatro alternativas ( $1=$ totalmente de acuerdo, $2=$ de acuerdo, $3=$ en desacuerdo, 4=totalmente en desacuerdo).

La validez del contenido del instrumento se calculó de acuerdo con los procedimientos recomendados por Kline (2000). El proceso de validación del instrumento en formato Likert incluyó la identificación de una muestra lo suficientemente adecuada para realizar el análisis de consistencia interna, usando como criterio el coeficiente alfa Cronbach (Kline, 1998). Se utilizó un índice de confiabilidad mayor o igual a 0.70 por ser el sugerido por Kline $(1998,2000)$. Esto implicó obtener por disponibilidad, para realizar la validación, una muestra de un mínimo de dos sujetos muestrales por cada reactivo (n total de 190 sujetos) debido a que el instrumento tenía 95 reactivos.

Referente al análisis estadístico, para todos los análisis cuantitativos se utilizó un nivel alfa de 0.05. Las características sociodemográficas de los participantes se calcularon a través de estadísticas descriptivas como: media, mediana, frecuencia y desviación estándar. Además de explorar las propiedades psicométricas de las escalas del instrumento, se realizó el análisis de factores exploratorios con rotación Varimax. Se evaluó también el índice de validez interna, utilizando el Alfa Cronbach, que fuera igual o mayor de 0.70 (Kline, 2005). Finalmente, el instrumento quedó compuesto de 21 reactivos y cinco componentes o factores. La validez de contenido del instrumento final de 21 reactivos alcanzó un Alfa Cronbach de 0.848, lo que es considerado como adecuado, según Kline (2000). Las cargas seleccionadas de los reactivos de los diferentes componentes son iguales o mayores a 0.40 . La escala dirigida a determinar las prácticas sexuales de los/as participantes no quedó incluida en el instrumento final debido a que al unirse al resto de los reactivos de los diferentes componentes debilitó su alfa.

La validez de contenido de cada componente también alcanzó índices adecuados. El componente identificado como «Influencia de las creencias religiosas y/o espirituales en la vida de los/as participantes» obtuvo una carga de 0.807 . De igual manera, los componentes: «Percepción de las consecuencias de tener relaciones sexuales»; «Impacto de las creencias religiosas 
y espirituales en las relaciones sexuales»; «Percepción de las relaciones sexuales por género»; e «Impacto de las relaciones sexuales en las prácticas espirituales y religiosas», alcanzaron cargas de: $0.747,0.804,0.927$ y 0.897 , respectivamente (Véase Tabla 1).

Tabla 1. Componentes, sus reactivos y respectivas cargas

\begin{tabular}{|c|c|c|c|}
\hline Nombre & Componente & Ítem & Carga \\
\hline \multirow{7}{*}{1} & \multirow{7}{*}{$\begin{array}{l}\text { Influencia de las creencias } \\
\text { religiosas y/o espirituales } \\
\text { en la vida de los/as } \\
\text { participantes }\end{array}$} & $\begin{array}{l}\text { III.A.5. Mis creencias religiosas } \\
\text { me ayudan a enfrentar mis } \\
\text { preocupaciones, enfermedades y } \\
\text { problemas. }\end{array}$ & .807 \\
\hline & & III.A.l. Dios ve nuestras acciones & .779 \\
\hline & & $\begin{array}{l}\text { III.A.6. Utilizo mis creencias } \\
\text { religiosas cuando tomo decisiones } \\
\text { importantes. }\end{array}$ & .702 \\
\hline & & $\begin{array}{l}\text { III.A.3. Trato de vivir mi vida de } \\
\text { acuerdo con mis creencias religiosas. }\end{array}$ & .606 \\
\hline & & $\begin{array}{l}\text { III.A.12. Es mejor evaluar las } \\
\text { situaciones del diario vivir usando las } \\
\text { normas morales de una religión. }\end{array}$ & .482 \\
\hline & & $\begin{array}{l}\text { III.A.10. Dependo de un Dios o un } \\
\text { Ser supremo para que me ayude a } \\
\text { resolver mis problemas. }\end{array}$ & .481 \\
\hline & & $\begin{array}{l}\text { IV.A.6 La forma mejor para evitar las } \\
\text { enfermedades de transmisión sexual } \\
\text { y los embarazos es la abstinencia. }\end{array}$ & .470 \\
\hline Total & & & .807 \\
\hline \multirow{5}{*}{2} & \multirow{5}{*}{$\begin{array}{l}\text { Percepción de las } \\
\text { consecuencias de tener } \\
\text { relaciones sexuales }\end{array}$} & $\begin{array}{l}\text { IV.A.2. Si tuviera relaciones sexuales, } \\
\text { haría que mi pareja perdiera el } \\
\text { respeto hacia mí. }\end{array}$ & .783 \\
\hline & & $\begin{array}{l}\text { IV.A.3. Tener relaciones sexuales } \\
\text { haría que mis amistades se } \\
\text { avergonzaran de mí. }\end{array}$ & .750 \\
\hline & & $\begin{array}{l}\text { V.1. Si tuviera relaciones sexuales me } \\
\text { sentiría culpable. }\end{array}$ & .605 \\
\hline & & $\begin{array}{l}\text { V.4. Mis creencias religiosas impiden } \\
\text { que tenga relaciones sexuales antes } \\
\text { de casarme. }\end{array}$ & .415 \\
\hline & & $\begin{array}{l}\text { IV.A.10. Las mujeres que tienen } \\
\text { relaciones sexuales antes del } \\
\text { matrimonio tienen una dudosa } \\
\text { reputación. }\end{array}$ & .414 \\
\hline Total & & & .747 \\
\hline
\end{tabular}

Alternativas. Cuadernos de Trabajo Social, 21, 2014, pp. 193-213 - ISSN 1133-0473 DOI: 10.14198/ALTERN2014.21.10 


\begin{tabular}{|c|c|c|c|}
\hline Nombre & Componente & Ítem & Carga \\
\hline \multirow{5}{*}{3} & \multirow{5}{*}{$\begin{array}{l}\text { Impacto de las creencias } \\
\text { religiosas y espirituales en } \\
\text { las relaciones sexuales }\end{array}$} & $\begin{array}{l}\text { V.3. Besarme y acariciarme con } \mathrm{mi} \\
\text { pareja es pecado. }\end{array}$ & .770 \\
\hline & & $\begin{array}{l}\text { V.10. Dios/Ser Supremo me castigaría } \\
\text { si tuviera relaciones sexuales antes de } \\
\text { contraer matrimonio. }\end{array}$ & .716 \\
\hline & & $\begin{array}{l}\text { V.2. Tener relaciones sexuales antes } \\
\text { del matrimonio es pecado. }\end{array}$ & .561 \\
\hline & & $\begin{array}{l}\text { V.6. Las relaciones sexuales antes del } \\
\text { matrimonio afectan mi relación con } \\
\text { Dios o Ser Supremo. }\end{array}$ & .552 \\
\hline & & $\begin{array}{l}\text { V.12. Para evitar tener relaciones } \\
\text { sexuales utilizo diferentes prácticas } \\
\text { espirituales como por ejemplo: } \\
\text { la oración, el ayuno, la lectura de } \\
\text { la Biblia, buscar consejería con } \\
\text { el Pastor/Sacerdote/Imán/Líder } \\
\text { religioso, etc. }\end{array}$ & .468 \\
\hline Total & & & .804 \\
\hline \multirow{2}{*}{4} & \multirow{2}{*}{$\begin{array}{l}\text { Percepción de las } \\
\text { relaciones sexuales por } \\
\text { género }\end{array}$} & $\begin{array}{l}\text { IV.A.13 Los adolescentes varones } \\
\text { pueden tener relaciones sexuales } \\
\text { libremente. }\end{array}$ & .898 \\
\hline & & $\begin{array}{l}\text { IV.A.14 Las adolescentes mujeres } \\
\text { pueden tener relaciones sexuales } \\
\text { libremente. }\end{array}$ & .889 \\
\hline Total & & & .927 \\
\hline Nombre & Componente & Ítem & Carga \\
\hline \multirow{2}{*}{5} & \multirow{2}{*}{$\begin{array}{l}\text { Impacto de las relaciones } \\
\text { sexuales en las prácticas } \\
\text { espirituales y religiosas }\end{array}$} & $\begin{array}{l}\text { V.9. Si tuviera relaciones sexuales } \\
\text { disminuiría mi tiempo de oración y } \\
\text { lecturas religiosas. }\end{array}$ & .880 \\
\hline & & $\begin{array}{l}\text { V.8. Si tuviera relaciones sexuales } \\
\text { disminuiría el número de veces que } \\
\text { asisto a la iglesia. }\end{array}$ & .853 \\
\hline Total & & & .897 \\
\hline
\end{tabular}

Fuente: Elaboración propia basada en los cuestionarios contestados.

\section{Procedimiento}

Una vez lograda la validez del instrumento, se administró a 251 estudiantes, los cuales fueron seleccionados por disponibilidad. Se acudió a los salones de clase donde se encontraban estos/as participantes y luego de una orientación sobre los pormenores del estudio se comenzó el proceso de reclutamiento. La orientación incluyó: los propósitos del estudio; las razones para estudiar el tema; el método de recopilación de los datos; la naturaleza voluntaria de su participación; el proceso de consentimiento y asentimiento para participar; la confidencialidad del proceso; los riesgos relacionados a su participación; la utilidad que tendrían los hallazgos para los/as profesionales de la conducta, el 
Departamento de Educación y otros escenarios profesionales en la intervención, prevención, tratamiento y educación de los/as adolescentes; así como en el surgimiento de nuevos estudios en el tema.

Bastó que uno solo de los padres o encargados legales autorizara la participación de su hijo/a en el estudio debido al riesgo mínimo que constituía para los/as participantes. La Hoja de Consentimiento Informado y Asentimiento del/la adolescente incluyó la firma del padre o la madre o el/la encargado/a legal y la del/la adolescente participante. Una copia de esta hoja le fue entregada a cada uno de los firmantes. La investigadora y su asistente, en el día y hora coordinada con los/as directores/as escolares y los/as maestros/as, entregaron los cuestionarios a los/as participantes y esperaron por su cumplimentación. No hubo tiempo límite para la contestación del cuestionario.

Debido a que la participación en el estudio fue voluntaria no se pudo garantizar la cantidad igual por género de participantes. Participar no contempló incentivo ni compensación alguna. Tampoco, existía relación alguna de los/as participantes con la investigadora.

Una vez finalizado el proceso de administración, los datos fueron ingresados en el programa IBM SPSS Statistics (Statistical Analysis in Social Science), versión 20 y se realizaron los análisis estadísticos pertinentes.

\section{Resultados}

El estudio comenzó explorando la experiencia religiosa y espiritual de los/as participantes a través de once preguntas cerradas. Se les proveyó la definición de espiritualidad y religión y características de las mismas, la que debían utilizar para indicar cómo se clasificaban a sí mismos/as: espirituales, religiosos/ as, ambos o ninguno de ellos. La definición de espiritualidad incluyó: (a) sentir una llenura interna, paz; (b) tener armonía y conexión con uno mismo, con otra gente, con el universo; (c) puede incluir creer en un dios o dioses o no creer en dios o dioses; (d) puede incluir tener una religión o no tenerla; (e) puede incluir asistir o no asistir a una iglesia o templo; $y$ (f) realizar prácticas de meditación, tener experiencias sagradas o místicas, el uso de rituales de sanación, cristales o pociones especiales y comunicación con los espíritus, etc. La religión se definió como: (a) creencias o prácticas de un grupo de gente organizada; (b) creencia en Dios o un Ser Supremo; (c) se relaciona con las instituciones de fe, o sea iglesias, templos, mezquitas, etc., doctrina religiosa y reglas espirituales; (d) realizar prácticas religiosas como: orar, rezar, ayunar, meditar, confesarse, sacrificios, etc.; y (e) se relaciona con la lectura de libros religiosos (por ejemplo: la Biblia, el Corán, etc.), música religiosa, reglas religiosas, participar en grupos religiosos, etc. 
Los/as participantes ( $\mathrm{n}=251)$, en su mayoría, son religiosos/as $(85.2 \%)$; solo $14.7 \%$ indicó no tener religión. Los participantes religiosos/as informaron que eran católicos (52.9\%) y protestantes/evangélicos (40.4\%). Más de tres cuartas partes (81.4\%) de los/as participantes se consideraron religiosos/ as y espirituales; y el $68.1 \%$ religioso/a. El $73.5 \%$ informó que asiste a la iglesia desde que nació y acuden a ella de una a tres veces a la semana (70.4\%). Un $24.2 \%$ lo hace una vez al mes. Para el $74.5 \%$ de los/as participantes, la religión es muy importante en sus vidas y para el $69.9 \%$, lo es la espiritualidad. El $62.5 \%$ pertenece al grupo de jóvenes de su iglesia, y el 9.2\% funge como líder de dicho grupo.

Los/as participantes religiosos/as y espirituales se involucran en diversidad de prácticas espirituales, las que consideran muy importantes. Más de la mitad informó que leen la Biblia; oran; rezan; asisten al rito de la Santa Cena; oran antes de comer, dormir, cuando tienen problemas y por los enfermos; ofrendan; hablan a otros sobre Dios; y escuchan música religiosa. Informaron que confían completamente en Dios y cuando oran, le piden perdón por cualquier pecado cometido. El 45.8\% expresó que busca apoyo espiritual del pastor o del sacerdote.

Influencia de las creencias religiosas y espirituales en la vida de los/as participantes

Los datos recopilados muestran que los/as participantes que indicaron no tener religión contestaron las preguntas de alto contenido religioso como por ejemplo, la de «Dios ve nuestras acciones». El $83.7 \%$ del total de los que no tenían religión estuvo de acuerdo (totalmente de acuerdo y de acuerdo) con esta aseveración. Esta disposición a responder estas preguntas, a pesar de no tener religión, puede obedecer a factores como: experiencia religiosa previa, alguna inclinación religiosa o a su percepción de lo que debe ser o hacer el/la que tiene religión.

Los hallazgos del estudio parecen respaldar que las creencias religiosas y espirituales ocupan un lugar importante en las conductas de los/as participantes. El estudio reveló que tres cuartas partes de los/as adolescentes participantes (74.5\%) tratan de vivir de acuerdo a sus creencias religiosas y las utilizan para tomar decisiones importantes (76.5\%). Además, les ayudan a enfrentar sus preocupaciones, enfermedades y problemas (84.9\%). La figura de Dios tiene preeminencia en su visión de mundo y de la vida (98.8\%) porque dependen de él para resolver sus problemas y piensan que este ve sus acciones (68.5\%). Cuando se indagó sobre la utilización de las normas morales de una religión para evaluar las situaciones del diario vivir, los/as no 
religiosos/as (32.4\%) se alejaron de los religiosos/as (58.9\%) en su respuesta. Una abrumadora mayoría de los/as participantes (80.5\%) apoyó la abstinencia sexual como la mejor forma de evitar las enfermedades de transmisión sexual y el embarazo.

\section{Creencias religiosas/espirituales y las relaciones sexuales}

Los hallazgos apuntan que las creencias religiosas desempeñan un papel importante en la conducta sexual de los/as participantes religiosos (Véase Tabla 2). Los/as participantes no religiosos, en una vasta mayoría, están en desacuerdo con las premisas que buscan medir esta relación. El 56.2\% de los/

Tabla 2 Influencia de la espiritualidad y la religión en las conducta sexuales

\begin{tabular}{|c|c|c|c|}
\hline Reactivos & $\mathrm{n}$ & $\begin{array}{c}\text { Totalmente de } \\
\text { acuerdo/de acuerdo }\end{array}$ & $\begin{array}{c}\text { Totalmente en } \\
\text { desacuerdo/Desacuerdo } \\
\end{array}$ \\
\hline $\begin{array}{l}\text { V.1. Si tuviera relaciones sexuales } \\
\text { me sentiría culpable. }\end{array}$ & 205 & 92 (44.9\%) & $113(55.1 \%)$ \\
\hline $\begin{array}{l}\text { V.2. Tener relaciones sexuales antes } \\
\text { del matrimonio es pecado. }\end{array}$ & 206 & $114(55.3 \%)$ & $92(44.7 \%)$ \\
\hline $\begin{array}{l}\text { V.3. Besarme y acariciarme con mi } \\
\text { pareja es pecado. }\end{array}$ & 203 & 19 (9.4\%) & $184(90.6 \%)$ \\
\hline $\begin{array}{l}\text { V.4. Mis creencias religiosas } \\
\text { impiden que tenga relaciones } \\
\text { sexuales antes de casarme. }\end{array}$ & 203 & $114(56.2 \%)$ & 89 (43.8\%) \\
\hline $\begin{array}{l}\text { V.6. Las relaciones sexuales antes } \\
\text { del matrimonio afectan mi relación } \\
\text { con Dios o Ser Supremo. }\end{array}$ & 204 & $94(46.1 \%)$ & $110(53.9 \%)$ \\
\hline $\begin{array}{l}\text { V.8. Si tuviera relaciones sexuales } \\
\text { disminuiría el número de veces que } \\
\text { asisto a la iglesia. }\end{array}$ & 204 & $19(9.3 \%)$ & 185 (90.7\%) \\
\hline $\begin{array}{l}\text { V.9. Si tuviera relaciones sexuales } \\
\text { disminuiría mi tiempo de oración y } \\
\text { lecturas religiosas. }\end{array}$ & 205 & $25(12.2 \%)$ & $180(87.8 \%)$ \\
\hline $\begin{array}{l}\text { V.10. Dios/Ser Supremo me } \\
\text { castigaría si tuviera relaciones } \\
\text { sexuales antes de contraer } \\
\text { matrimonio. }\end{array}$ & 202 & $50(24.8 \%)$ & $152(75.2 \%)$ \\
\hline $\begin{array}{l}\text { V.12. Para evitar tener relaciones } \\
\text { sexuales utilizo diferentes prácticas } \\
\text { espirituales como por ejemplo: } \\
\text { la oración, el ayuno, la lectura de } \\
\text { la Biblia, buscar consejería con } \\
\text { el Pastor/Sacerdote/Imán/Líder } \\
\text { religioso, etc. }\end{array}$ & 207 & $93(44.9 \%)$ & $114(55.1 \%)$ \\
\hline
\end{tabular}

Fuente: Elaboración propia basada en los cuestionarios contestados.

Alternativas. Cuadernos de Trabajo Social, 21, 2014, pp. 193-213 - ISSN 1133-0473

DOI: 10.14198/ALTERN2014.21.10 
as participantes religiosos/as están de acuerdo (totalmente de acuerdo y de acuerdo) con que sus creencias religiosas les impiden tener relaciones sexuales antes del matrimonio y si las tuvieran, no disminuiría el número de veces que asistirían a la iglesia (90.7\%). Para un poco más de la mitad de los/as participantes religiosos/as tener relaciones sexuales antes de contraer matrimonio es pecado (55.3\%). Solo $44.9 \%$ se sentiría culpable si las tuvieran y el $24.8 \%$ piensa que Dios los/as castigaría. Prácticas religiosas como la oración, el ayuno, la lectura de la Biblia, buscar consejería con el pastor, sacerdote o líder religioso/a son usadas por el $44.4 \%$ para evitar tener relaciones sexuales premaritales.

De acuerdo al 53.9\% de los/as participantes religiosos, incurrir en relaciones sexuales no afectaría su relación con Dios. Tampoco provocaría que disminuyera su tiempo de adoración y lecturas religiosas (87.8\%) ni el número de veces que asiste a la iglesia (90.7\%). Igualmente, no afectaría el respeto que su pareja tiene hacia ellos/as (72.5\%) ni sus amistades se avergonzarían de ellos/as (81.8\%).

Comparación de la conducta sexual de los/as adolescentes por afiliación religiosa

El desbalance de la muestra, en el que 37 estudiantes no son religiosos y 214 lo son, impidió el análisis correlacional propuesto de los dos grupos y con ello, la prueba de las hipótesis. No obstante, se pudieron establecer relaciones entre las afiliaciones religiosas (católica y protestante/evangélica) de los/ as participantes. Para ello se realizó un análisis no paramétrico a través de la prueba ji cuadrada. Los resultados revelaron diferencias estadísticamente significativas $(p<.05)$ de los católicos en relación con los protestantes/evangélicos en: su percepción de las relaciones sexuales; el impacto de sus creencias religiosas en las relaciones sexuales; y en el impacto de las prácticas religiosas en las relaciones sexuales. Los reactivos que registraron diferencias fueron los siguientes: «Si tuviera relaciones sexuales me sentiría culpable» $\left(\chi^{2}=23.655\right.$, $p<.003)$; «Tener relaciones sexuales premaritales antes del matrimonio es pecado» $\left(\chi^{2}=18.946, p<.001\right)$; «Las relaciones sexuales antes del matrimonio afectan mi relación con Dios» $\left(\chi^{2}=41.485, p<.000\right)$; «Para evitar tener relaciones sexuales utilizo diferentes prácticas religiosas como por ejemplo: la oración, el ayuno, la lectura de la Biblia, buscar consejería espiritual con el/la Pastor/a/ Sacerdote/Imán/Líder religiosos/a» $\left(\chi^{2}=25.285, p<.001\right) ;$ «Si tuviera relaciones sexuales disminuiría mi tiempo de oración y lecturas religiosas» $\left(\chi^{2}\right.$ $=21.490, p<.000)$ y «Si tuviera relaciones sexuales disminuiría el número de veces que asisto a la iglesia» $\left(\chi^{2}=41.485, p<.000\right)$. 
Cuando se examinó la relación entre protestantes/evangélicos y católicos se encontraron diferencias estadísticamente significativas $(p<.05)$ en las premisas que determinaban la influencia de las creencias religiosas en sus vidas. Las premisas fueron: «Trato de vivir mi vida de acuerdo con mis creencias religiosas» $\left(\chi^{2}=19.322, p<.001\right)$; «Utilizo mis creencias religiosas cuando tomo decisiones importantes» $\left(\chi^{2}=17.247, p<.002\right)$; «Dependo de un Dios o un ser supremo para que me ayude a resolver mis problemas» $\left(\chi^{2}=17.940, p<.001\right)$; $\mathrm{y}$ «Es mejor evaluar las situaciones del diario vivir usando las normas morales de una religión» $\left(\chi^{2}=13.550, p<.035\right)$.

Respecto a la percepción e impacto de sus creencias religiosas en las relaciones sexuales los/as protestantes/evangélicos/as se diferenciaron de sus homólogos en: «Mis creencias religiosas impiden que tenga relaciones sexuales antes de casarme» $\left(\chi^{2}=28.198, p<.000\right)$ y «Dios/Ser Supremo me castigaría si tuviera relaciones sexuales antes de contraer matrimonio» $\left(\chi^{2}=11.187, p\right.$ $<.025)$.

Los/as participantes católicos/as y protestantes/evangélicos/as no se diferenciaron estadísticamente en ocho reactivos $(p<.05)$. Es decir, su denominación religiosa no los distanció en lo que piensan respecto a las siguientes premisas: «Dios ve nuestras acciones» $\left(\chi^{2}=11.358, p<.182\right)$; «La forma mejor de evitar las enfermedades de transmisión sexual y los embarazos es la abstinencia» $\left(\chi^{2}=1.147, p<.997\right)$; «Si tuviera relaciones sexuales, haría que mi pareja perdiera el respeto hacia mí» $\left(\chi^{2}=10.076, p<.260\right)$; «Tener relaciones sexuales haría que mis amistades se avergonzaran de mí» $\left(\chi^{2}=6.334, p<.610\right)$; $\mathrm{y}$ «Besarme y acariciarme con mi pareja es pecado» $\left(\chi^{2}=7.754, p<.458\right)$.

En lo que respecta al género, este pareció no variar las respuestas de los/as participantes de ambos grupos en los 21 reactivos del instrumento. En aquellos que se hizo una clara alusión al género como: «Los adolescentes varones pueden tener relaciones sexuales libremente» $\left(\chi^{2}=7.340, p<.500\right)$; «Las adolescentes mujeres pueden tener relaciones sexuales libremente» $\left(\chi^{2}=4.943, p\right.$ $<.764) ; \mathrm{y}$ «Las mujeres que tienen relaciones sexuales antes del matrimonio tienen una dudosa reputación» $\left(\chi^{2}=11.949, p<.154\right)$, no registraron diferencias estadísticas significativas.

\section{Discusión}

Las hipótesis de esta investigación anticiparon que existían diferencias estadísticamente significativas $(p<.05)$ en las conductas sexuales de los/as participantes religiosos/as y los/as no religiosos/as; entre los valores de la escala la Influencia de la Espiritualidad en las Conductas Sexuales; el involucramiento en actividades sexuales sustitutivas a la relación coital; y por género en sus 
conductas sexuales. La participación desproporcionada de los sujetos sin religión, en comparación con los que indicaron ser religiosos en la muestra, imposibilitó la comparación válida entre los dos grupos. Aunque esta situación trae limitaciones a los propósitos del estudio, es también necesario considerarlo como un hallazgo importante. La selección de estudiantes de escuelas estatales en el estudio buscaba variedad de ideologías espirituales y religiosas, no obstante, nos topamos con que el $85.2 \%$ eran religiosos cristianos. De acuerdo a un estudio reciente del Pew Research Center's Forum on Religion \& Public Life (NoticiaCristiana.com, 2014), Puerto Rico, de los 3, 725,789 habitantes que posee, 3, 630,000 son creyentes cristianos (2, 620,000 católicos y 940,000 protestantes). Así pues, aunque un/a adolescente haya indicado que no tiene religión o sea practicante de alguna, no lo/a exime de haber tenido alguna experiencia en ella por influencia directa (familia) o indirecta (escuela, amistades). Esta explicación respalda otro de los hallazgos: los/as participantes que se autodenominaron no religiosos/as contestaron las preguntas de alto contenido religioso. Esto sugiere que, para futuras investigaciones sobre el tema, haya procedimientos más rigurosos y criterios bien específicos de selección de estos dos grupos, que garanticen su representación.

Los resultados del estudio, desde el marco del conglomerado de participantes (religiosos y no religiosos), respaldan el rol de la religión como factor proscriptor e inhibidor de las relaciones sexuales antes del matrimonio, lo que es cónsono con las múltiples investigaciones realizadas sobre el tema (Burdette y Hill, 2009; Farmer, Trapnell y Meston, 2008; Haglund y Fehring, 2010; Hardy y Raffaelli, 2003; Morales Alejandro, 2014; Noden, Gomes y Ferreira, 2010; Rotosky, Regnerus y Comer, 2003; Sinha, Cnaan y Gelles, 2007; Spearman, 2006. Los resultados parecen mostrar el compromiso de los/as religiosos/as con sus creencias religiosas cuando piensan que Dios ve sus acciones; asisten varias veces a la semana a las actividades de su iglesia; se identifican como religiosos y espirituales; y realizan diversas prácticas religiosas. Las creencias religiosas actúan como mecanismos que les ayudan a enfrentar sus dificultades, enfermedades y preocupaciones, y además, son el marco de referencia que utilizan para tomar decisiones importantes.

Los/as católicos/as y protestantes/evangélicos/as dan importancia a sus creencias religiosas y expresan compromiso hacia ellas, asistiendo a la iglesia varias veces a la semana a las actividades que ofrecen, realizando las prácticas espirituales correspondientes a su doctrina y desempeñando varios roles como miembros de la congregación religiosa. La gran mayoría se consideró religioso/a y espiritual, percibiendo su religiosidad fusionada a la espiritualidad. Este hallazgo es claramente compatible con la investigación de Morales 
Alejandro (2014: 71), sobre la influencia de la religión y la espiritualidad en 36 adolescentes puertorriqueños/as religiosos/as. Estos/as adolescentes concibieron la espiritualidad como el nivel más alto de religiosidad, en la que «el Espíritu de Dios está dentro del cuerpo de la persona como una señal y la guía a comportarse correctamente... demanda que esta no se contamine, porque tiene que ser pura y limpia». Esta conexión es alimentada por prácticas espirituales como: orar/rezar, ayunar, lectura de la Biblia y asistencia a misa o servicios religiosos, las que también están presentes en los/as participantes.

Los resultados sugieren que la prominencia religiosa interviene en sus conductas sexuales. Cónsono con los resultados del estudio de Burdette y Hill (2009), las prácticas religiosas de los/las adolescentes de orar, rezar y leer la Biblia estuvieron relacionadas a la forma de evitar las relaciones sexuales. Aunque piensan que Dios ve sus acciones, a solo la mitad de los/as participantes religiosos/as les impide tener relaciones sexuales antes del matrimonio; y menos de esta, se sienten culpables si incurrieran en ellas. Los/as participantes se están involucrando en relaciones sexuales premaritales o en otras actividades sexuales, independientemente del compromiso que informaron tener con sus creencias religiosas.

Esta inconsistencia en conductas: sexual y religiosa, se ha observado en otros estudios científicos. Burdette y Hill (2009) encontró que los/as adolescentes cristianos/as, a pesar de la asociación fuerte de la prominencia religiosa con la demora o retraso de las relaciones sexuales, se involucraban en otras actividades sexuales. Al igual que Rotosky, Regnerus y Comer (2003) también se descubrió que la religiosidad impacta parcialmente la conducta sexual de los/as adolescentes. Estos hallazgos manifiestan la disminución del control social de la religión en las conductas sexuales en los/as adolescentes religiosos/as puertorriqueños/as. Parecen estar apelando más al racionalismo y a su autodeterminación. Las opiniones morales de los/as adolescentes del estudio reflejan el propio cuestionamiento de sus decisiones morales, propio de su etapa de desarrollo. En el razonamiento moral dirigido a las decisiones sexuales, además del contexto espiritual, están presentes otros contextos sociales en los/as que son socializados/as, el que incluye las amistades. Las amistades de los/as participantes no se avergonzarían de ellos/as si incurrieran en las relaciones sexuales, lo que parece ser un respaldo a su conducta.

Hardy y Rafaelli (2003) opinan que la contradicción de los adolescentes religiosos entre lo que creen y cómo se comportan puede provocarles angustia e inducirlos/as a alterar sus creencias religiosas, incluyendo abandonarlas. Sin embargo, en el caso de los/as participantes, alejarse o abandonar la religión no ha sido una opción. Más bien, se considera que es la visión positiva que tienen 
de Dios, la que parece mitigar o eliminar las posibles consecuencias espirituales de su conducta sexual. De acuerdo a Morales Alejandro (2014), los/as adolescentes religiosos/as protestantes y católicos/as puertorriqueños/as describen a Dios con atributos como: protector; ayuda a resolver los problemas; se puede hablar con Él y contarle todo; perdonador; nunca los/as deja, aunque «caigan»; y no los/as abandonará ni se alejará de ellos/as, los que pueden ser parte de sus percepciones. También pueden estar ayudándolos/as a manejar sus sentimientos de culpa y de pecado cuando se involucran en relaciones sexuales prematrimoniales. Las prácticas religiosas de la oración, el ayuno, las lecturas bíblicas y la asistencia frecuente a la iglesia también fungen como mecanismos de afrontamiento.

Por otro lado, las diferencias significativas encontradas entre las afiliaciones religiosas católicas y protestantes/evangélicas dejaron de manifiesto lo que Remez (1990) y Ogland e Hinojosa (2012) argumentan respecto al rol de las denominaciones religiosas en las actitudes hacia el sexo premarital. Similar a sus hallazgos, se halló que las afiliaciones protestantes tienden a ser más restrictivas en el sexo premarital en comparación con los/as adolescentes de otras afiliaciones religiosas.

Los resultados de este estudio develan el rol de la religiosidad y la espiritualidad en las conductas sexuales de los/as adolecentes puertorriqueños/as. Estos hallazgos deben interpretarse desde varias limitaciones. Primero, los datos son obtenidos a través de un diseño transversal, por lo que, aunque son capaces de señalar las relaciones fuertes entre las afiliaciones religiosas, el género y los 21 reactivos del instrumento, no pueden realizarse inferencias causales; tampoco generalizaciones más grandes a la población de adolescentes de 15 a 18 años de edad puertorriqueños/as.

La falta de representatividad de los/as adolescentes que no tienen religión y las implicaciones que acarreó la comparación con sus homólogos/as religiosos/as, requiere que se tomen acciones y establezcan procedimientos específicos en la selección de la muestra para que se obtenga representatividad de ambos grupos. Asimismo, en la construcción del instrumento, sería muy útil precodificar la categoría protestante y otras religiones que permitiera a los/ as participantes establecer distinciones entre ellas. Se observó que algunos/ as participantes no supieron ubicarse o clasificarse en las denominaciones indicadas y solo se limitaron a indicar que eran cristianos/as. La exigencia del Departamento de Educación respecto a los/as estudiantes que podían participar, también limitó la amplitud y diversidad de la muestra.

A pesar de estas limitaciones, se cree que este estudio contribuye significativamente a la investigación de la intersección de la religión y las conductas 
sexuales de los/as adolescentes puertorriqueños/as. Una de sus principales contribuciones es el desarrollo y validación de un instrumento pionero, novel, validado con adolescentes puertorriqueños sobre las conductas sexuales (IEIRCS: VLMA). Del mismo modo, aporta a la incipiente producción de conocimiento basado en evidencia sobre la influencia de la espiritualidad y religiosidad en la conducta de los/as adolescentes, y abre las puertas para ampliar las investigaciones existentes y generar otras sobre el tema. Asimismo, pretende promover que los/as trabajadores/as sociales tomen en cuenta las diversas expresiones de la religión y la espiritualidad de sus clientes y comunidades, y adquieran destrezas para su integración ética en el proceso de exploración e intervención profesional.

\section{Agradecimientos}

La investigadora agradece el apoyo económico del Decanato de Estudios Graduados e Investigación de la Universidad de Puerto Rico en la realización de este estudio. Además, se reconoce la colaboración del Dr. José Rodríguez Gómez como consultor estadístico. Igualmente, a la trabajadora social, Danalyz Pizarro, asistente de investigación, por su compromiso y esfuerzo durante todo el proceso de investigación; y a la Dra. Gisela Negrón Velázquez, directora del Departamento de Trabajo Social, por el respaldo ofrecido.

\section{Bibliografía}

BARRET, M. J. (2003). Healing from trauma: The quest for spirituality. In Walsh, F. (Ed.). Spiritual resources in family therapy (pp. 193-208). New York: The Guilford Press.

BECVAR, D. S. (1998). Soul healing and the family. En D. S. Becvar (Eds.), The family, spirituality and social work. Nueva York: The Haworth Press, Inc.

BurdetTe, A. y HiLl, T. (2009). Religious Involvement and Transitions into Adolescent Sexual Activities. Sociology of religion, 70(1), 28-48.

CANDA, E. R. (1998). Spirituality sensitive social work: Key concepts and ideals. Recuperado de http//www.bemidjistate.edu/academics/publications/social_ work_journal/issues01/articles/canda.html

COMPTE-SPONVILle, A. (2006). El alma del ateísmo: Introducción a una espiritualidad sin Dios. Barcelona: Paidós.

DavidSOn SR., J. K., MoORE, N. B., EARLE, J. R., y Davis, R. (Summer 2008). Sexual attitudes and behavior at four universities: Do region, race, and/or religion matter? Adolescence, 43(170), 189-217. 
FARMer, M. A., Trapnell, P. D., y Meston, C. M. (2008). The relation between sexual behavior and religiosity subtypes: A test of the secularization hypothesis. Springer Science Business Media LLC 2008

HAGLund, K.A. y FeHring, R. J. (2010). The association of religiosity, sexual education, and parental factors with risky sexual behaviors among adolescents and young adults. Journal of Religion and Health, 49(4), 460-472. Doi 10. 1007/s 10943-009-9267-5.

HugEN, B. (2001b). Spirituality and religion in social work practice: A conceptual model. En M. Van Hook, B. Hugen \& M. Aguilar (Eds.), Spirituality within religious traditions in social work practice (pp. 1-5). California: Brooks/COLE.

HARDY, S. y RAFFAELLI, M. (2003). Adolescent religiosity and sexuality: An investigation of reciprocal influence. Journal of Adolescence, 26, 731-739.

Hernández SAmpieri, R., Fernández Collado, C., y Baptista lucio, P. (1998). Metodología de la investigación. México: McGraw-Hill Interamericana Editores, S. A. de C. V.

Hutchinson, E. D. (2008). Dimensions of human behavior: Person and environment. California: Sage Publications, Inc.

KuIne, P. (1998). The New Psychometrics Science, Psychology and Measurement. New York: Routledge.

KLINE, P. (2000). The handbook of psychological testing. New York: Routledge.

KLINE, T. (2005). Psychological testing: A practical approach to design and evaluation. Thousand Oaks, CA: Sage Publications.

Knight, J. D., Sherritt, L., Harris, S. K., Holder, D. W., Kulig, J, Shrier, L. A., et al.(2007, April). Southern Medical Journal, 100 (4), 349-355.

KOENIG, H. G. (2008). Medicine religion and health: Where science and spirituality meet. Pensilvania: Templeton Foundation Press.

LAwsHE, C. H. (1975). A quantitative approach to content validity. Personnel Psychology, 28, 563-575.

Morales Alejandro, L. I. (2014). Espiritualidad y religión: Sus influencias en las conductas de riesgo- uso de drogas, alcohol y sexualidad temprana- en niños/as $y$ adolescentes. San Juan: Isla Negra Editores.

Murray-Swank, N. A., Pargament, K. I., y Mahoney, A. (2005). At the crossroads of sexuality and spirituality: The sanctification of sex by college students. International Journal for the Psychology of Religion, 15(3), 199-219. doi:10.1207/s15327582ijpr1503_2.

NODEN, B.H., GOMES, A. y FERREIRA, A. (2010). Influence of religious affiliation and education on HIV knowledge and HIV-related sexual behaviors among unmarried youth in rural central Mozambique. Aids Care, 10, 1285-1294. doi: $10.1080 / 09540121003692193$.

NoticiaCristiana.com. (2014, 2 de septiembre). En Puerto Rico el 97\% son cristianos asegura un reciente estudio. Recuperado de http://www.noticiacristiana. 
com/ciencia_tecnologia/estudios/2012/01/en-puerto-rico-el-97-son-cristianos-asegura-un-reciente-estudio.html

Ogland, C. P. y Hinojosa, P. (2012). Religion and social attitudes: Examining the contours of religion in moral judgments toward premarital sex and cohabitation in contemporary Brazil. Sociology of Religion, 73(4), 411-428. doi:10.1093/socrel/srs027

Papalia, D. E., Olds, S., y Feldman, R. (2010). Desarrollo humano. México: McGraw-Hill Interamericana.

PARGAMENT, K. I. (1997). The psychology of religion and coping: Theory, research, practice. Nueva York: The Guilford Press.

PEO EARly, B. (1998). Between two worlds: The psychospiritual crisis of a dying adolescent. En E. R. Canda, (Ed.), Spirituality in social work: New directions (pp. 67-81). Nueva York: The Haworth Pastoral Press.

Remez, L. (1990). Adolescents' Attitudes Toward Premarital Sex Affect Religious Activity. Family Planning Perspectives, 22(1), 41-42.

Rotosky, S., Regnerus, M., y Comer, W. (2003). Coital debut: The role of religiosity and sex attitudes in the Add Health Survey. The Journal of Sex Research, 40(4), 358-367.

SinHA, J. W., CNAAM, R. A., y RiCHARD, J. (2007). Adolescent risk behaviors and religion: Findings from a national study. Journal of Adolescence, 30, 231-249.

SPEARMAN, D. R. (2006). Impact of Christianity and God-mediated locus of control on adolescent risky behaviors. Tesis doctoral no publicada, University of Hartford, Connecticut. Recuperado de books.google.com.pr/ books?id=kKXxYtwAACAAJ

Uecker, J. E., Angotti, N., y Renerur, M. D. (2008). Going most of the way: «Technical virginity» among American adolescents. Social Science Research, 37, 1200-1215.

WALSH, F. (2003). Religion and spirituality: Wellsprings for healing and resilience. En F. Walsh (Ed.), Spiritual resources in family therapy (pp. 3-27). Nueva York: The Guilford Press. 